\title{
Dimensión crítica de la reescritura académica. Análisis textométrico en un curso de formación docente en lenguaje*
}

\section{Critical Dimension}

of Academic

Rewriting.

Textmetric Analysis

in a Language

Teacher Training

Course

\author{
Dimensão crítica \\ da reescrita \\ acadêmica. Análise \\ textométrica em um \\ curso de formação \\ de professores em \\ linguagem
}

Giohanny Olave Arias ${ }^{* *}$ Orcid: https://orcid.org/ 0000-0001-6794-6472

Una versión preliminar de este artículo fue presentada en el Ix Congreso Internacional de la Cátedra Unesco para el mejoramiento de la calidad y la equidad en la educación, con base en la Lectura y la Escritura para América Latina, del 23 al 27 de julio, en la Universidad de Cuenca, Ecuador. El trabajo fue presentado en coautoría con el profesor Héctor Fabio Ramírez, de la Universidad Tecnológica de Pereira.

** Doctor en Lingüística. Profesor de planta en la Escuela de Idiomas de la Universidad Industrial de Santander.

Correo electrónico: giolavar@uis.edu.co

(c) (1) \$

Para citar este artículo

Olave, G. (2019). Dimensión crítica de la reescritura académica. Análisis textométrico en un curso de formación docente en lenguaje.

Folios, 50, 41-50.

doi: 10.17227/Folios.50-10175

Artículo recibido

$30 \cdot 07 \cdot 2018$

Artículo aprobado $25 \cdot 01 \cdot 2019$ 


\section{Resumen}

En este artículo reportamos resultados iniciales de una investigación en curso cuyo objetivo es analizar los modos de aparición de imaginarios sociales sobre la lectura y la escritura, en los textos producidos al inicio de la formación de profesores de lengua materna. Dentro de un diseño metodológico cuasiexperimental longitudinal, utilizamos herramientas de la textometría (análisis Reinert o de mundos lexicales y análisis factorial de correspondencias) para comparar las respuestas que escribieron y reescribieron estudiantes de primer semestre, al inicio y al final de un curso de comprensión y producción textual, frente a la pregunta: “¿Por qué estudiar la Licenciatura en Español y Literatura, en 'tiempos de crisis'?". En los resultados, interpretamos las autorreformulaciones en las reescrituras desde sus modificaciones en las redes de significados en torno a la profesión docente. Concluimos que los nuevos vínculos de significados que aparecen en las reescrituras de los estudiantes dan cuenta de una integración incipiente de preocupaciones políticas al pensar la enseñanza de la lectura y la escritura. El camino que hemos emprendido avanza de una alfabetización académica hacia una alfabetización política en la formación de docentes de lengua materna.

\section{Palabras clave}

textometría; reformulación textual; alfabetización académica; formación docente; pensamiento político

Abstract

In this paper we report the initial results of an ongoing research whose objective is to analyze the modes of appearance of social imaginaries about reading and writing, in the texts produced at the beginning of the training of teachers of Spanish language and literature. Within a longitudinal quasi-experimental methodological design, we use textometry tools (Reinert analysis or lexical worlds and factorial correspondence analysis) to compare the answers written and rewritten by first semester students, at the beginning and at the end of a course of comprehension and textual production, related to the question Why study the Degree in Spanish and Literature, in "times of crisis"? In the results, we interpret the self-reformulations in the rewritings from their modifications in the networks of meanings around the teaching profession. We conclude that the new meaning links that appear in the student's rewritings show an incipient integration of political concerns when thinking about the teaching of reading and writing. The path we have taken advances from academic literacy to political literacy in the training of teachers of Spanish language and literature.

\section{Keywords}

textometry; textual reformulation; academic literacy; teacher training; political thinking

\section{Resumo}

Este artigo apresenta os resultados iniciais de uma investigação em curso cujo objetivo é analisar os modos de ocorrência do imaginário social sobre a leitura ea escrita nos textos produzidos no início da formação de professores de língua materna. Dentro de um projeto longitudinal, estudo quasi-experimental, usamos ferramentas da textometría (análise Reinert ou mundos lexicais e análise fatorial de correspondência) para comparar as respostas que escreveram e reescreveram os estudantes do primeiro semestre no início e final de um curso de compreensão e produção textual, diante da pergunta: "Por que estudar o grau em espanhol e literatura, em 'tempos de crise'?". Nos resultados, interpretamos as auto-reformulações nas reescritas a partir de suas modificações nas redes de significados em torno da profissão docente. Concluímos que os novos links de significado que aparecem nas reescritas dos estudantes revelam uma integração incipiente de preocupações políticas ao pensar sobre o ensino da leitura e da escrita. 0 caminho que percorremos avança da alfabetização acadêmica para a alfabetização política na formação de professores de língua materna.

\section{Palavras-chave}

textometria; reformulação textual; alfabetização acadêmica; formação de professores; pensamento político 


\section{Introducción}

En esta propuesta, utilizamos herramientas de la textometría (Pincemin, 2010; Salem y Fleury, 2009) para interpretar un conjunto de reescrituras de textos producidos al inicio de la formación de profesores de lengua materna, en la Licenciatura en Español y Literatura de la Universidad Tecnológica de Pereira, Colombia. Esas reescrituras fueron producto de una intervención didáctica que hizo énfasis en la dimensión crítica y sociocultural de los textos académicos; por tal razón, la motivación principal de esta iniciativa es la orientación de los estudios sobre alfabetización y literacidad académicas (Carlino, 2013) hacia una alfabetización política desde los primeros niveles de formación de docentes, en los currículos y espacios institucionalizados de prácticas formativas letradas.

Dentro del activo campo de los estudios sobre enseñanza de la lectura y la escritura universitarias en Latinoamérica (Ávila, 2017; Navarro et al., 2016; Parodi, 2016; Pontificia Universidad Javeriana, 2017) y en Colombia (Cisneros, Olave y Rojas, 2013; Rojas, Olave y Cisneros, 2016; González y Vega, 2013; Rojas, 2017; entre otros), también se ha atendido a la reescritura en contextos didácticos universitarios (Arnoux, 2009; Álvarez, García y Qués, 2010; Muñoz, Massi y Liendo, 2016; Pereira y DiStefano, 2009; Pereira y Valente, 2014; Valente, 2013, 2016). Sin embargo, predomina en ellos la evaluación de estrategias de reformulación resuntiva (habilidad para resumir) e intradiscursiva (paráfrasis y mecanismos de reiteración, explicación, argumentación y conexión entre segmentos dentro del mismo texto). En menor medida se ha explorado la reescritura de textos completos realizada por el mismo autor en formación, con reformulaciones no parafrásticas (Rossari, 1997; Roulet, 1987) y con introducción de focos y temas nuevos a partir del texto ya producido.

En cuanto al tratamiento de la reescritura como operación discursiva más allá de la corrección estilística y en una vertiente no normativa de los borradores de escritura, asumimos la posición de Jitrik (2000, pp. 85-88), retomada y profundizada empíricamente por Valente $(2013,2016)$ y Pereira y Valente (2014). Los textos que son reescritos por el mismo autor contienen las huellas de operaciones cognitivas y discursivas mediadoras entre concepciones y puntos de vista, algunos no previstos en la escritura inicial, que se reconfiguran a través de diversos procesos de autorreformulación: "Por tal motivo, la reescritura, en la que entran en juego cuestiones conceptuales e interpretativas, es el resultado de una nueva representación semántica que enriquece la inicial" (Valente, 2016, p. 139). En ese punto reside la diferencia entre reescribir y revisar o corregir los textos propios; operaciones que suelen presentarse como formas idénticas de nombrar una de las etapas finales de la escritura en los modelos procesuales de producción textual:

El objeto de las operaciones de reescritura es distinto, pues se vincula semánticamente con la construcción del sentido, con cuestiones conceptuales e interpretativas. Dicha operación es vista, entonces, como una sobreescritura porque se trata de escribir en un segundo momento. (Pereira y Valente, 2014, p. 200).

La clave aquí es dejar de ver la reescritura como una producción de enmiendas, reparaciones inmediatas o ajustes al plan inicial que realiza el autor a sus propios textos. En cambio, las mediaciones pedagógicas y las reflexiones teóricas que ocurren entre la escritura y la reescritura pueden quedar integradas en esta última, en diferentes grados y apropiaciones, a partir de las decisiones que tome el autor en esa segunda instancia de producción textual.

Para una aproximación inicial a este objeto, proponemos analizar contrastivamente el nivel léxico-semántico de las escrituras y reescrituras, desde una perspectiva textométrica. Este enfoque pretende avanzar más allá de la cuantificación de unidades léxicas, ligadas a la lexicometría, hacia la puesta en texto de esas cuantificaciones, particularmente en cuanto a su contexto de emergencia y a las relaciones intertextuales que establecen. El análisis, pues, no se limita a la descripción estadística del léxico, sino que se interesa por la interpretación del texto en su complejidad, con una gestión cualitativa englobante; así, "lo cuantitativo guía el análisis, pero 
es un examen cualitativo (con retorno al texto) el que determina la interpretación" (Pincemin, 2010, p. 43).

\section{Metodología}

Dentro de un diseño metodológico cuasiexperimental longitudinal, hablamos de dimensión crítica de la reescritura académica para referirnos a las reformulaciones textuales que los estudiantes realizaron bajo una doble demanda: incorporar en sus textos saberes acerca del problema de lo crítico en el pensamiento crítico latinoamericano, central en el material didáctico (Rojas y Olave, 2015) de las actividades de la clase que cursaron; y asumir una perspectiva autocrítica frente a su propia escritura, para reescribir un texto que escribieron al iniciar el curso "Taller de lectura y escritura I”, impartido en junio de 2017 en 48 horas clase, distribuidas en 4 horas diarias.

El curso se enfocó en el acompañamiento sistemático del estudio de un texto académico-científico prototípico. La guía didáctica de ese acompañamiento incluyó tareas en torno al capítulo "En búsqueda de un nuevo paradigma crítico", incluido en el libro Conocer desde el Sur, de Boaventura de Sousa Santos (2006). Se trabajaron cinco dimensiones analíticas (textual, intertextual, enunciativa, valorativa y sociocultural) (ICFEs, 2013, 2014) a través de talleres de comprensión y producción textual. Dentro de este último aspecto, se le propuso como examen final a cada estudiante la reescritura crítica de la respuesta que escribió al iniciar el curso, frente a la siguiente pregunta: “¿Por qué estudiar la Licenciatura en Español y Literatura en 'tiempos de crisis'?".

Presentaremos sintéticamente una interpretación delos resultados obtenidos, a partir de la comparación entre las versiones inicial y final de 20 textos escritos y reescritos como respuesta a esa pregunta abierta. Los resultados fueron obtenidos desde el software para análisis textométrico Iramuteq (versión 0,7 alpha 2), específicamente a partir del índice de coocurrencia basado en la prueba chi cuadrado, que permite realizar un análisis de similitudes léxicos y un análisis factorial de correspondencias $(\mathrm{AFC})$, además de visualizar los resultados gráficamente en dendogramas y planos cartesianos, respectivamente.
En el primer tipo de análisis, se identifican los mundos lexicales (Reinert, 1993); un método que permite rastrear el enfoque compartido por un grupo heterogéneo de textos, a través de las huellas que dejan los puntos de vista de los diferentes autores en los discursos, anclados a sus elecciones léxicas. Se trata de la organización de grupos de lexías en torno a núcleos de significado; las similitudes y diferencias entre usos léxicos permiten constituir categorías conceptuales, que son presentadas como hipótesis de interpretación. La clasificación de las lexías se realiza en orden jerárquico descendente, según los resultados de la prueba chi cuadrado, que calcula la correlación entre variables, comparando la distribución observada de los datos con la distribución esperada de ellos. Las categorías se muestran en dendogramas y representan agrupaciones de puntos de vista de los estudiantes al responder la pregunta abierta planteada.

El AFC se concentra en la probabilidad de aparición simultánea de lexías. La coincidencia entre ellas indica cercanías entre significados, con las cuales se constituyen agrupamientos y se confirman las categorías emergentes del análisis Reinert. Estos grupos se acercan o se alejan de otros grupos de lexías coocurrentes, todo lo cual ayuda a identificar las asociaciones semánticas que realizan los informantes. En este nivel analítico del léxico se pueden confirmar o afinar hipótesis interpretativas sobre el direccionamiento de las asociaciones semánticas y el tratamiento de los temas sobre los cuales se les ha pedido escribir a los informantes.

\section{Resultados}

\section{Análisis de mundos lexicales}

Las figuras 1, 3 y 2, 4, escrituras y reescrituras, respectivamente, permiten visualizar la organización de los agrupamientos lexicales, de acuerdo con los mundos de referencia y los puntos de vista que los configuran. 


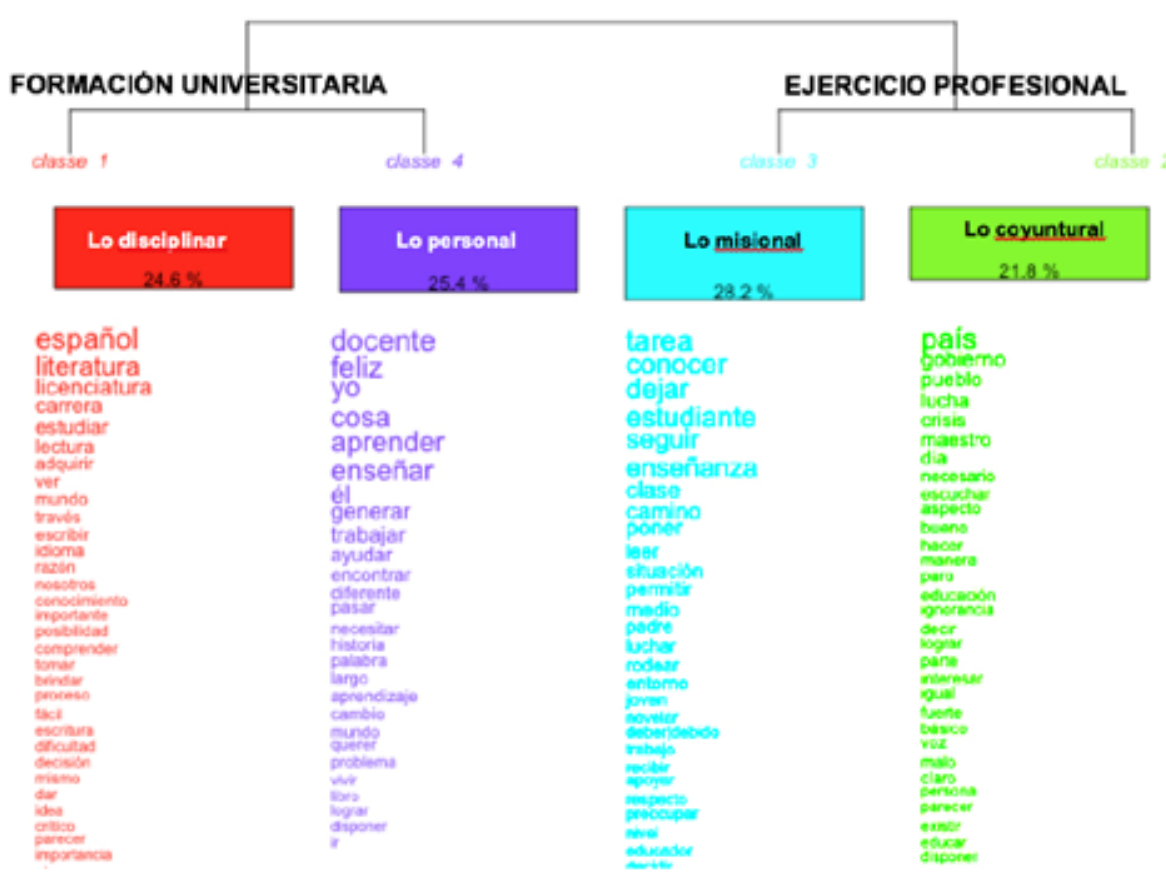

Figura 1. Dendograma de mundos lexicales en la fase de escritura

Fuente: elaboración propia.

En su primera escritura, los estudiantes configuran dos mundos lexicales independientes: la formación universitaria que les ofrece su carrera (clases 1 y 4 ), por un lado, y el ejercicio profesional del docente (clases 2 y 3), por otro. La distribución porcentual de estos ejes de significado es muy equilibrada (50\%) y refiere la presencia constante de sus tópicos en el conjunto de escrituras. En tal medida, la separación de estos mundos traduce una representación general escindida entre la universidad y el trabajo, esto es, entre las representaciones de dos roles sociales (el estudiante y el docente) que, en esta etapa inicial de su formación, se tensionan en la visión que revelan los estudiantes sobre sí mismos, en tanto que futuros docentes.

En el caso del mundo de la formación universitaria, un 24,6\% (clase 1, en rojo) de unidades léxicas se asocia con los significados de lo disciplinar o los saberes académicos de la carrera que cursan, frente a un $25,4 \%$ (clase 4 , en violeta) que activa significados de lo personal, como las motivaciones particulares de los autores para estudiar esta carrera, sus trayectorias de vida y la afirmación de subjetividades. En el caso del mundo del ejercicio profesional, domina un $28,2 \%$ (clase 3 , en turquesa) de significados agrupados en torno a la representación de lo misional de la labor docente, esto es, la función social ejercida desde la profesión particular; en el mismo mundo lexical, pero con una incidencia menor $(21,8 \%$, clase 2 , en verde), se visualiza el ejercicio profesional desde las problemáticas de coyuntura política, a propósito de un cese de actividades docentes y movilizaciones públicas llevadas a cabo por los profesores de las escuelas y colegios del país, durante 37 días, desde el 16 de mayo de 2017 (Semana.com, 2017).

El agrupamiento de significados que introducen el tema político coyuntural en el momento de la escritura de estos textos aparece aislado del mundo de la formación universitaria. Este agrupamiento representa el porcentaje más bajo de las clases segmentadas, es decir, con menor protagonismo en la elaboración de sus respuestas, pero además, cuando es incorporado, lo coyuntural es reconocido por los estudiantes como una problemática del ejercicio profesional que está más cercana a los aspectos 
misionales o vocacionales de la labor, que al mundo institucional de la universidad y de los procesos formativos que los tocan más directamente.

En sus textos, los estudiantes se posicionan frente a la dimensión política de la labor docente desde el lugar de los espectadores, tras la barrera de la formación universitaria construida como saberes disciplinares y trayectorias de vida personal. La configuración de estos mundos lexicales puede leerse en clave de un cierto privilegio de las motivaciones individuales sobre las colectivas, cuando se interroga acerca de la elección de ser docente de lengua materna: la construcción de los significados de lo social presenta mayor dispersión que lo asociado con la formación universitaria. Esa mayor o menor cohesión entre los significados puede visualizarse en un gráfico de dispersión a partir del análisis factorial de correspondencias (AFC) (figura 2):

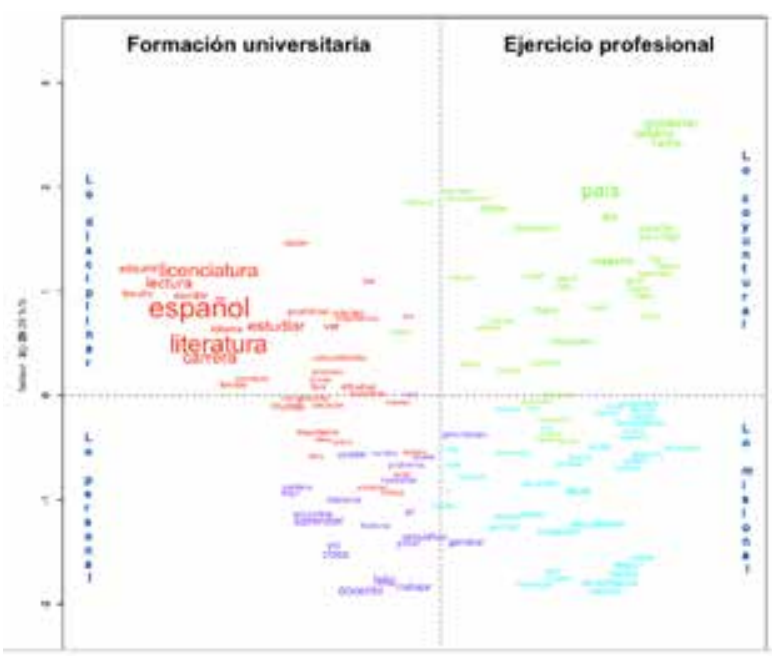

Figura 2. Análisis factorial de correspondencias en la fase de escritura

Fuente: elaboración propia.

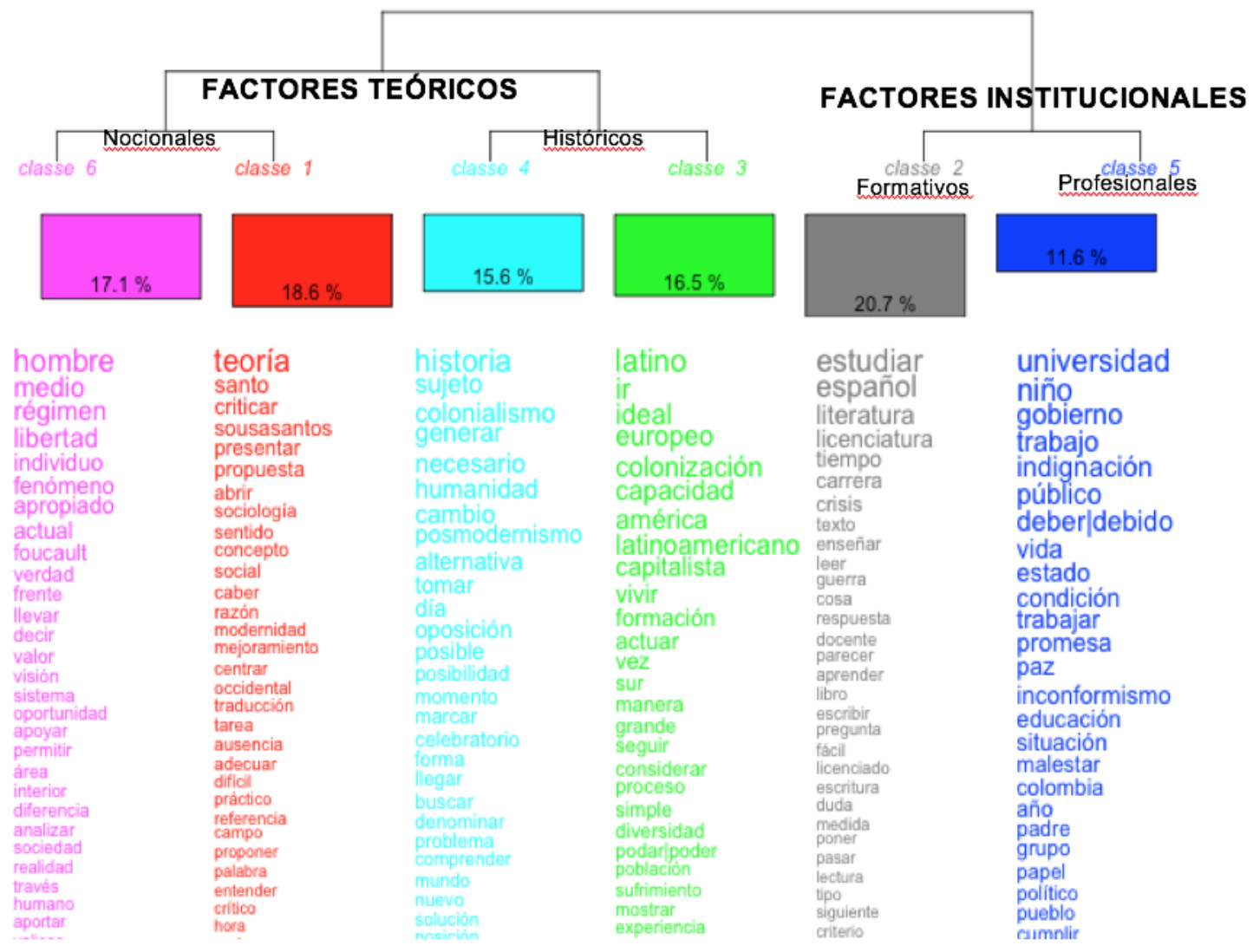

Figura 3. Dendograma de mundos lexicales en la fase de reescritura

Fuente: elaboración propia. 
Como vemos, la disyunción entre los mundos de la formación universitaria y el ejercicio profesional muestra una mayor cohesión en el cuadrante de lo disciplinar y dispersión más evidente en los demás, sobre todo en el espacio de lo coyuntural. En este último, los significados asociados gobierno, pueblo y lucha (en verde), activados en función del evento contextual del paro de maestros, aparecen como el subconjunto más aislado y equidistante de la dimensión más personal o subjetiva de la carrera docente (en color magenta).

En síntesis, resaltamos que en esta primera respuesta a la pregunta “ ¿Por qué estudiar la Licenciatura en Español y Literatura en 'tiempos de crisis'?", quienes inician su formación docente convergen en una visión despolitizada de la preparación universitaria y de la dimensión vocacional de la docencia, lo cual se extiende a la reducción de los saberes sobre la literatura y la lingüística dentro de un ámbito disciplinar más teórico que práctico y más cognoscitivo que social. Esto no significa que en los textos no aparezcan tematizados asuntos políticos, especialmente los coyunturales, sino que ellos son introducidos y tratados de manera dispersa y desligada de lo vocacional (lo personal más lo misional de la docencia).

A partir de esta configuración de mundos lexicales, utilizada como una escritura diagnóstica, las reescrituras presentan una configuración distinta de mundos lexicales, como lo muestra el dendograma de la figura 3.

La escisión radical entre formación universitaria y ejercicio profesional desaparece en las reescrituras para conformar, ahora yuxtapuestas, un nuevo mundo lexical que identificamos bajo la categoría de factores institucionales. En el agrupamiento de los factores formativos (clase 2, en gris: 20,7\%) se aglutinan los elementos disciplinares y las motivaciones personales de la elección de la carrera profesional, para aproximarlos a las cuestiones más directamente ligadas con la institución universitaria y con los problemas políticos coyunturales, es decir, con lo que denominamos factores institucionales (clase 5, en azul: 11,6\%), pues activan en los estudiantes la tematización y reflexión acerca de los órdenes sociales establecidos y sus fenómenos políticos derivados.

Un segundo mundo lexical aparece también en estas reescrituras. Hemos rotulado como factores teóricos el conjunto de dos agrupamientos de significados que comparten un esfuerzo por incluir y dar cuenta de las herramientas conceptuales ofrecidas durante el desarrollo del curso. Estos factores se dividen, a su vez, en nocionales (clases 1 y 6 , en rojo $-18,6 \%$ - y magenta $-17,1 \%-$, respectivamente) e históricos (clases 3 y 4 , en verde $-16,5 \%$ - y turquesa $-15,6 \%-$, respectivamente), de acuerdo con su mayor énfasis en la abstracción de los contenidos o en su inserción/presentación dentro de la trama histórica.

El predominio porcentual de los factores teóricos sobre los institucionales $(68,7 \%$ sobre $32,3 \%)$ puede leerse como un síntoma de la dificultad para integrar los saberes movilizados en clase con los saberes sociales de referencia. No obstante, este mayor índice también está influido por los requisitos del género textual (parcial o examen final) como género de formación académica (Natale y Stagnaro, 2014), en el cual la demanda de explicitar los aspectos teóricos puede llegar a aislarlos de su uso aplicado e interpretativo sobre la realidad, como el profesor lo esperaría. En cuanto a la distribución espacial de las correspondencias, la tendencia de los factores teóricos es al aglutinamiento o mayor cohesión entre los significados, en contraste con una dispersión más evidente en los factores institucionales y especialmente en los profesionales (figura 4).

En contraste con los resultados del AFC en la fase de escritura, en las reescrituras los mundos lexicales aparecen menos cerrados y más permeables a la articulación, como se aprecia en el eje 0 que marca la división vertical entre ambos agrupamientos. Aunque los significados principales de la clase 5 (color violeta) se muestran más bien aislados y equidistan de los factores históricos, otros que forman parte de esta clase se aproximan a la nube de lexías más compacta, en el rango de - 1 a 0 . Las herramientas analíticas utilizadas en este primer abordaje no permiten evaluar la imbricación de lo teórico y lo empírico en estas reescrituras, pero sí evidencian que, frente a esta 
demanda textual, los estudiantes logran cohesionar con mayor densidad los significados teóricos que los referidos a los factores institucionales. En este caso, leemos el contraste entre dispersión y cohesión en las correspondencias léxicas en clave de articulación o desarticulación entre los mundos lexicales construidos y sus agrupamientos afines.

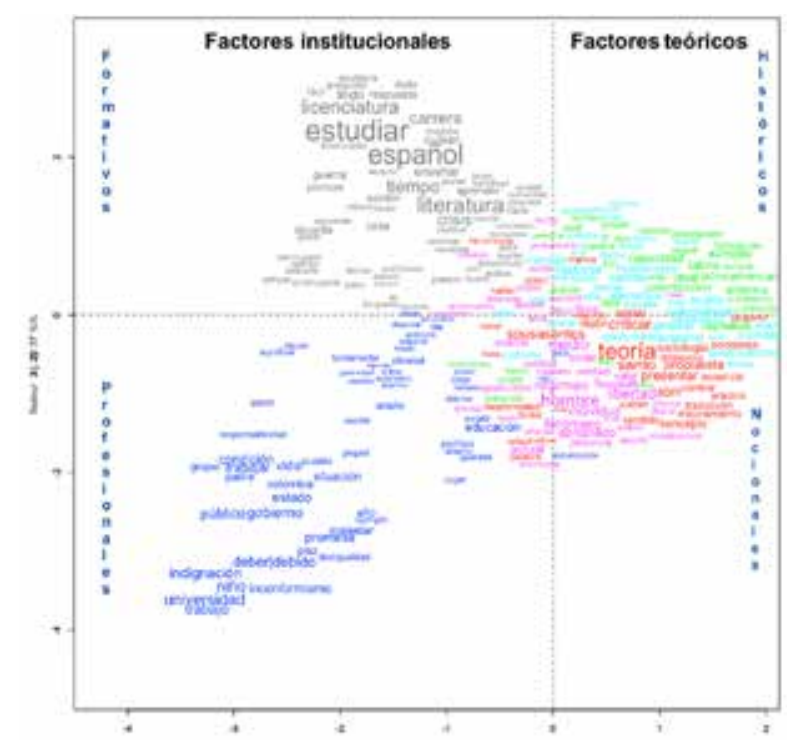

Figura 4. Análisis factorial de correspondencias en la fase de reescritura

Fuente: elaboración propia.

\section{Conclusiones}

En este artículo hemos sintetizado una interpretación sobre el contraste entre respuestas a la pregunta “Por qué estudiar la Licenciatura en Español y Literatura en 'tiempos de crisis'?" Los textos fueron escritos por estudiantes de primer semestre de esta carrera, al iniciar y finalizar un taller de lectura y escritura concentrado en la comprensión de un texto inserto en el pensamiento crítico latinoamericano.

La dimensión crítica de la reescritura, como ejercicio en las aulas de formación universitaria, presenta dos aristas complementarias: la primera tiene que ver con el contenido crítico de los textos que los profesores hacemos circular en el aula, es decir, no solo con su género textual sino también con su pertinencia textual, como apuntábamos en un trabajo anterior (Rojas, Olave y Cisneros, 2016, p. 241). Teniendo en cuenta esta primera arista, concluimos que el acompañamiento a la lectura del texto de De Sousa Santos (2006) se resuelve en la incorporación en las reescrituras de una mirada más problematizadora y focalizada acerca de lo crítico en relación con la docencia del español y la literatura. Una segunda dimensión apunta hacia lo autocrítico implicado al reescribir un texto de autoría propia. Frente a esa demanda y contando con el texto escrito originalmente, todos los estudiantes introdujeron modificaciones y construyeron un texto nuevo para dar respuesta a la misma pregunta inicial. La arista autocrítica se manifestó en las diversas autorreformulaciones que aparecieron en las reescrituras y que aquí abordamos, acotada e inicialmente, desde los nuevos vínculos entre significados.

Aunque de manera incipiente y solo en lo referido a la coocurrencia de significados, las segundas versiones de los textos conformaron reescrituras críticas al articular lo formativo y lo profesional en la problemática de lo institucional. Con este mismo criterio, encontramos que el establecimiento de relaciones para la construcción de sentidos presenta más dificultades al tratar de articular los factores teóricos con los institucionales, es decir, al utilizar directamente la fundamentación teórica proporcionada para asumir una postura propia frente a las condiciones institucionales de la formación de docentes en el país. En suma, resaltamos el ejercicio de reformulación de los textos originales, orientado durante el desarrollo de la asignatura pero elaborado de forma autónoma en el momento final de la reescritura, pues muestra las huellas de un primer redimensionamiento político de la lectura y la escritura en la construcción de significados coocurrentes.

La aproximación textométrica aquí presentada puede verse como una fase inicial dentro del estudio de reescrituras en contextos didácticos preocupados por ese redimensionamiento político de la alfabetización universitaria. La continuidad de este análisis apuntará al abordaje de otros niveles, como el retórico y el argumentativo.

La perspectiva asumida en este documento acerca de la formación de docentes de lengua materna 
sostiene que es en el temprano reconocimiento y trabajo sobre su componente político donde los profesores pueden ejecutar transformaciones deseables colectivamente para sus grupos sociales. El espacio formativo en lectura y escritura, especialmente al inicio de la formación de docentes, tiene en la politización de su profesión una tarea ineludible para llenar de pertinencia la alfabetización académica y trascenderla hacia una alfabetización política, de modo que contribuya con la comprensión del productor de textos como sujeto político y de los textos mismos como acciones urgentes en el medio donde se interactúa.

Esa postura está anclada al ámbito de influencia del profesor y al abordaje de las problemáticas cotidianas que aparecen en su escuela y comunidad particulares. No se trata, pues, de una dimensión política en abstracto de la lectura y la escritura, en relación con valores universales o necesariamente partidarios, sino de la concreción de su potencialidad (la capacidad política de la toma de la palabra) en el entorno inmediato en que los jóvenes se preparan para enseñar a leer y escribir.

\section{Agradecimientos}

Este trabajo fue posible gracias al espacio curricular ofrecido por el director de la Escuela de Español y Literatura de la Universidad Tecnológica de Pereira, Arbey Atehortúa Atehortúa, y por la colaboración activa del profesor Héctor Fabio Ramírez. Un sentido agradecimiento también para cada uno de los estudiantes del curso, nuestros futuros profesores, quienes cada día siguen buscando respuestas más profundas a la pregunta de por qué ser docentes de lengua materna en Colombia.

\section{Referencias}

Álvarez, G., García, M. y Qués, M. (2010). Entornos virtuales de aprendizaje. Una propuesta para mejorar las habilidades de reformulación productiva de estudiantes preuniversitarios. Educación, Comunicación, Tecnología, 5(9), 1-24.

Arnoux, E. (dir.) (2009). Pasajes: escuela media-enseñanza superior: propuesta en torno a la lectura y la escritura. Buenos Aires: Biblos.
Ávila, N. (2017). Postsecondary writing studies in Hispanic Latin America: Intertextual dynamics and intellectual influence. London Review of Education, 15(1), 21-37. DOI: https://doi.org/10.18546/ LRE.15.1.03

Carlino, P. (2013). Alfabetización académica diez años después. Revista Mexicana de Investigación Educativa, 18(57), 355-381.

Cisneros, M., Olave, G. y Rojas, I. (2013). Alfabetización académica y lectura inferencial. Bogotá: Ecoe.

De Sousa Santos, B. (2006). Conocer desde el Sur. Para una cultura política emancipatoria. Lima, Perú: Universidad Nacional Mayor de San Marcos (UnMsM).

González, B. y Vega, V. (2013). Lectura y escritura en la educación superior colombiana: herencia y deconstrucción. Interacción, 12, 195-201.

Instituto Colombiano para el Fomento de la Educación Superior (ICFEs) (2013). Módulo de Lectura Crítica Saber Pro 2013-2. Bogotá.

Instituto Colombiano para el Fomento de la Educación Superior (ICFEs) (2014). Módulo de Comunicación Escrita Saber Pro 2014-1. Bogotá.

Jitrik, N. (2000). Los grados de la escritura. Buenos Aires: Manantial.

Muñoz, R., Massi, M. y Liendo, J. (2016). La reformulación y su rol en el desarrollo de la competencia académica universitaria. Palabra, 5, 70-83.

Natale, L. y Stagnaro, D. (2014). El parcial presencial. En: F. Navarro (coord.), Manual de escritura para carreras de humanidades (pp. 103-134). Buenos Aires: FiloUBA.

Navarro, F., Ávila, N., Tapia, M., Cristovão, V., Moritz, M., Narváez, E. y Bazerman, Ch. (2016). Panorama histórico y contrastivo de los estudios sobre lectura y escritura en educación superior publicados en América Latina. Signos. Estudios de Lingüística, 49(S1), 100-126. DOI: 10.4067/S0718-09342016000400006

Parodi, G. (2016). Procesos de lectura y escritura en el mundo contemporáneo de lengua española. Signos. Estudios de Lingüística, 49(S1), 4-6.

Pereira, C. y DiStefano, M. (2009). Representaciones acerca de la tesis doctoral en las reescrituras de la Introducción. Un estudio de caso. En E.N. de Arnoux (dir.), Escritura y producción del conocimiento en carreras de posgrado (pp. 203-219). Buenos Aires: Santiago Arcos. 
Pereira, M. C. y Valente, E. (2014). De la revisión a la reescritura: recorridos de las producciones escritas de estudiantes universitarios. Revista Enunciación, 19(2), 199-214.

Pincemin, B. (2010). Semántica interpretativa y textometría. Tópicos del Seminario, 23, 15-55.

Pontificia Universidad Javeriana (UniJaveriana) (18 de abril de 2017). Conferencias Writing Research across Borders (WRAB) 2017 [Archivo de video]. Recuperado de https://www.youtube.com/playlist? list $=$ PL7oPhRcTBSbxAkiEASaKvN7vKW8UvuxEA

Reinert, M. (1993). Les “mondes lexicaux" et leur logique à travers l'analyse statisque d'un corpus de récits de cauchemars. Langage et Societé, 66, 5-39.

Rojas, I. (2017). Enseñar a leer y escribir en las disciplinas. Estado de la cuestión en las universidades colombianas. Folios, 45, 29-49.

Rojas, I. y Olave, G. (2015). Didáctica de la lectura y la escritura en la universidad. Material interno de cátedra. Universidad Tecnológica de Pereira.

Rojas, I., Olave, G. y Cisneros, M. (2016). Alfabetización académica y pedagogía de género discursivo en la lingüística sistémico-funcional. Una experiencia de trabajo. Signos, Estudios de Lingüística, 49(S1), 224-246.
Rossari, C. (1997). Les opérations de reformulation. Berna: Peter Lang.

Roulet, E. (1987). Complétude interactive et connecteurs reformulatifs. Cahiers de Linguistique Française, 11, 111-140.

Salem, A. y Fleury, S. (dir.) (2009). Explorations textométriques. París: Université de la Sorbonne Nouvelle Paris 3.

Semana.com (6 de junio de 2017 6). El ABC del paro docente. Recuperado de http://www.semana.com/ educacion/articulo/fecode-por-que-los-maestrosentraron-en-paro/527601

Valente, E.(2013). La definición del tema en trabajos monográficos en el inicio de los estudios de posgrado. Reescritura, reformulación y construcción del ethos. (Trabajo de grado de Especialización). Facultad de Filosofía y Letras, Universidad de Buenos Aires, Argentina.

Valente, E. (2016). Reescritura, reflexión teórica y argumentación en el nivel de posgrado. Traslaciones, 3(6), 135-164. 